\title{
Guest editorial: special issue on multi-robot and multi-agent systems
}

\author{
Nora Ayanian ${ }^{1} \cdot$ Paolo Robuffo Giordano ${ }^{2} \cdot$ Robert Fitch $^{3} \cdot$ Antonio Franchi $^{4,5} \cdot$ Lorenzo Sabattini $^{6}$
}

Published online: 19 February 2020

(c) Springer Science+Business Media, LLC, part of Springer Nature 2020

In the same way that computers have revolutionized the way we deal with information, robotics will enable a similar revolution in the way we operate in the real world. Future robots will form a backbone of pervasive, efficient networks of taskable agents-but, crucially, these will be systems of communicating, cooperative devices. Already, multiple robot and multiple agent systems (MRS/MAS) have shown their worth in fairly structured and semi-structured domains such as logistics, cargo management, and agriculture. Far more is on the immediate horizon: vehicular networks for transportation, security and surveillance, food supply, environmental monitoring and ecological impact assessment, services for manufacturing and assistance in rescue settings.

This special issue presents contributions that describe state-of-the-art results in distributed robotics and multi-agent systems research as applicable to autonomous robot systems. Topics represented include fundamental system capabilities including communication, data fusion, estimation, localisation, planning, and decision making; interactions between

\footnotetext{
Robert Fitch

rfitch@uts.edu.au

Nora Ayanian

ayanian@usc.edu

Paolo Robuffo Giordano

prg@irisa.fr

Antonio Franchi

a.franchi@utwente.nl

Lorenzo Sabattini

lorenzo.sabattini@unimore.it

1 University of Southern California, Los Angeles, CA 90089, USA

2 CNRS, Univ Rennes, Inria, IRISA, 35042 Rennes, France

3 University of Technology Sydney, Ultimo, NSW 2007, Australia

4 Robotics and Mechatronics Lab, Faculty of Electrical Engineering, Mathematics, and Computer Science, University of Twente, Enschede, The Netherlands

5 CNRS, LAAS-CNRS, Toulouse, France

6 University of Modena and Reggio Emilia, 42121 Reggio Emilia, Italy
}

humans and robot teams; and system tasks such as coverage, mapping, and tracking. Contributed papers were selected from a total of 37 submissions solicited via an open call for papers.

The issue opens with the topic of effective communication, which is fitting in that this is an enabling capability for many systems and algorithms. In their paper "Multi-robot online sensing strategies for the construction of communication maps", authors Alberto Quattrini Li et al. describe how a team of robots can efficiently map an area in terms of where two robots may communicate, in order to facilitate subsequent system deployment.

The next four papers consider challenges in estimation and data fusion for a team of robots connected by a communication network. The paper "Efficient recursive distributed state estimation of hidden Markov models over unreliable networks" by Amirhossein Tamjidi, Reza Oftadeh, Suman Chakravorty, and Dylan Shell presents a recursive filter for distributed state estimation on hidden Markov models that is suited to unreliable networks. In "Cooperative visual-inertial sensor fusion: fundamental equations and state determination in closed-form", authors Agostino Martinelli, Alessandro Renzaglia, and Alexander Oliva consider the problem of cooperative localisation and present a closed-form solution for cooperative visual/inertial sensor fusion. Ruofei Ouyang and Bryan Kian Hsiang Low present methods for cooperative perception in large-scale environments in their paper "Gaussian process decentralized data fusion meets transfer learning in large-scale distributed cooperative perception." Vivek Shankar Varadharajan, David St-Onge, Bram Adams and Giovanni Beltrame present a method for large-scale data transfer in distributed robot systems in "SOUL: data sharing for robot swarms."

Continuing on the topic of localisation, the paper "A robust localization system for multi-robot formations based on an extension of a Gaussian mixture probability hypothesis density filter" by Alicja Wasik, Pedro U. Lima, and Alcherio Martinoli proposes a method for cooperative state estimation that is effective in cluttered environments with significant measurement and communication uncertainty. Steven van der 
Helm, Mario Coppola, Kimberly N. McGuire, and Guido C. H. E. de Croon demonstrate cooperative localisation from range-only observations in their paper "On-board rangebased relative localization for micro air vehicles in indoor leader-follower flight."

A number of articles in the issue consider challenges in path planning, decision making, and control. The paper "dRRT*: Scalable and informed asymptotically-optimal multi-robot motion planning" by Rahul Shome et al. extends the dRRT algorithm, a sampling-based approach for multirobot motion planning, to allow for stronger theoretical guarantees. Jingin Yu presents an efficient algorithm for high-quality multi-robot motion planning on grids in the paper "Average case constant factor time and distance optimal multi-robot path planning in well-connected environments." From the perspective of reinforcement learning, authors Aaron Ma, Michael Ouimet, and Jorge Cortés introduce a hierarchical Markov decision process framework for satisfying spatially distributed objectives in large environments in their paper "Hierarchical reinforcement learning via dynamic subspace search for multi-agent planning."

The paper "Hybrid planning and distributed iterative repair for multi-robot missions with communication losses" by Patrick Bechon, Charles Lesire, and Magali Barbier presents a distributed algorithm for online repair of existing plans in the face of robot failure, varying objectives, and unreliable communication. Authors Pablo Gómez Esteban et al. also study decision making in a long-term context in their paper "Competition and cooperation in a community of autonomous agents," and describe methods for adapting to competitive or cooperative environments dynamically. Michael Otte, Michael J. Kuhlman, Donald Sofge study the task allocation problem in "Auctions for multi-robot task allocation in communication limited environments," and present a principled analysis of common auction algorithms that yields general insights for improving performance in the case of uncertain communication.

Rich tasks that move beyond typical geometric goal specifications are explored in the paper "Automated synthesis of decentralized controllers for robot swarms from highlevel temporal logic specifications" by Salar Moarref and Hadas Kress-Gazit, who propose a formal specification language to describe both team and individual behaviours and associated methods for automatically constructing control algorithms. An interactive approach for top-down control of multi-robot systems is presented by Valeria Villani et al. in the paper "Humans interacting with multi-robot systems: a natural affect-based approach", where the authors demonstrate a physical interface whereby the system adapts to the user's observed affective state. Masoume M. Raeissi and Alessandro Farinelli also address the properties and limitations of human operators in "Cooperative Queuing Policies for Effective Scheduling of Operator Intervention," and present an algorithmic method that allows for efficient use of human intervention in large-scale system deployments.

The final five papers in the issue focus on common classes of tasks performed by multi-robot systems in practice. Area coverage in the absence of localisation is studied by authors Marzieh Varposhti, Vesal Hakami, and Mehdi Dehghan, who present a distributed learning algorithm with convergence guarantees in the paper "Distributed coverage in mobile sensor networks without location information." The paper "CARE: Cooperative Autonomy for Resilience and Efficiency of robot teams for complete coverage of unknown environments under robot failures" by Junnan Song, Shalabh Gupta presents an algorithm for area coverage in the presence of robot failures.

Target tracking with an unknown and dynamic number of targets is explored in the paper "Distributed multi-target search and tracking using the PHD filter" by author Philip M. Dames, who integrates a distributed formulation of the Probability Hypothesis Density (PHD) filter into an algorithmic framework that is effective in systems with a large number of robots and targets. Target tracking in the context of the art gallery problem is studied by Guillermo J. Laguna and Sourabh Bhattacharya in the paper "Adaptive target tracking with a mixed team of static and mobile guards: deployment and activation strategies," which presents a collection of algorithms for various combinations of stationary and mobile robots. The final paper of the issue, "Air-ground cooperative topometric mapping of traversable ground" by Brice Renaudeau, Ouiddad Labbani-Igbida, and Gilles Mourioux, studies the problem of constructing a traversability map using both aerial and ground robots, and presents a hybrid topolog$\mathrm{ical} /$ metric graph-based approach along with experimental validation.

The special issue arose in part from the first IEEE International Symposium on Multi-Robot and Multi-Agent Systems (MRS), an initiative of the IEEE RAS Technical Committee on Multi-Robot Systems. The issue is edited by the Conference Chair, Editorial Board Editor-in-Chief, and Program Chairs of MRS 2017. All articles have been peer-reviewed in accordance with the high standards of Autonomous Robots.

The editors would like to thank the organizers and participants of MRS 2017 for their help in laying the groundwork on which this special issue is built. We would also like to thank all authors and reviewers who dedicated their time, effort, and ideas. Finally, we thank the Springer editorial staff who helped us along the way, and the journal's Editor-in-Chief who supported us wholeheartedly.

Publisher's Note Springer Nature remains neutral with regard to jurisdictional claims in published maps and institutional affiliations. 